\title{
Expression and Role of Voltage-Gated Sodium Channels in Human Dorsal Root Ganglion Neurons with Special Focus on Nav1.7, Species Differences, and Regulation by Paclitaxel
}

\author{
Wonseok Chang ${ }^{1,2}$ - Temugin Berta ${ }^{1,3}$ - Yong Ho Kim ${ }^{1,4} \cdot$ Sanghoon Lee $^{3}$. \\ Seok-Yong Lee ${ }^{5} \cdot$ Ru-Rong Ji ${ }^{1,6}$
}

Received: 13 January 2017/Accepted: 8 March 2017/Published online: 19 April 2017

(C) The Author(s) 2017. This article is an open access publication

\begin{abstract}
Voltage-gated sodium channels (Navs) play an important role in human pain sensation. However, the expression and role of Nav subtypes in native human sensory neurons are unclear. To address this issue, we obtained human dorsal root ganglion (hDRG) tissues from healthy donors. PCR analysis of seven DRG-expressed Nav subtypes revealed that the hDRG has higher expression of Nav1.7 ( $\sim 50 \%$ of total Nav expression) and lower expression of Nav1.8 $(\sim 12 \%)$, whereas the mouse DRG has higher expression of Nav1.8 ( $\sim 45 \%)$ and lower expression of Nav1.7 ( 18\%). To mimic Nav regulation in chronic pain, we treated hDRG neurons in primary cultures with paclitaxel $(0.1-1 \mu \mathrm{mol} / \mathrm{L})$ for $24 \mathrm{~h}$. Paclitaxel increased the Nav1.7 but not Nav1.8 expression and also increased the
\end{abstract}

Wonseok Chang, Temugin Berta and Yong Ho Kim have contributed equally to this study.

Temugin Berta

temugin.berta@uc.edu

$\triangle$ Ru-Rong Ji

ru-rong.ji@duke.edu

1 Department of Anesthesiology, Duke University Medical Center, Durham, NC 27710, USA

2 Department of Physiology and Biophysics, College of Medicine, Eulji University, Daejeon, Korea

3 Pain Research Center, Department of Anesthesiology, University of Cincinnati Medical Center, Cincinnati, OH 45267, USA

4 Department of Physiology, College of Medicine, Gachon University, Incheon, Korea

5 Department of Biochemistry, Duke University Medical Center, Durham, NC 27710, USA

6 Department of Neurobiology, Duke University Medical Center, Durham, NC 27710, USA transient $\mathrm{Na}^{+}$currents and action potential firing frequency in small-diameter $(<50 \mu \mathrm{m}) \mathrm{hDRG}$ neurons. Thus, the hDRG provides a translational model in which to study "human pain in a dish" and test new pain therapeutics.

Keywords Dorsal root ganglion - Neuropathic pain · Paclitaxel · Voltage-gated sodium channels

\section{Introduction}

Over the last 20 years, great progress has been made in identifying novel pain targets and illustrating the neuronal, immune, and glial mechanisms of pathological pain in animal models [1-4]. Despite the progress in preclinical studies and substantial investment by the pharmaceutical industry, little progress has been made in developing novel, efficacious, and safe analgesics for human pain conditions $[5,6]$. A translational gap from rodents to humans has been blamed for the failure in developing pain medications $[5,6]$. To address this issue, several groups including our own have begun to investigate pain mechanisms in native primary sensory neurons in human dorsal root ganglia (hDRGs) obtained from donors [7-11]. Davidson et al. demonstrated that a high proportion of hDRG neurons respond to the algogens allyl isothiocyanate and ATP, as well as the pruritogens histamine and chloroquine [7]. Importantly, hDRG neurons also respond to the inflammatory mediators bradykinin and prostaglandin E2, showing evidence of peripheral sensitization [7].

Voltage-gated sodium (Nav) channels play a critical role in the initiation and propagation of action potentials in excitable cells such as neurons and are the major targets of analgesics [12-14]. Nine subtypes of Nav (Nav1.1Nav1.9) have been identified; each subtype plays a distinct 
physiological role and also exhibits distinct expression patterns in DRG neurons. For example, Nav1.7-Nav1.9 are mainly expressed in small nociceptive neurons in DRGs [12, 15-19]. In particular, recent genetic studies have demonstrated a critical role of Nav1.7 in pain sensation in humans. Loss-of-function mutations in SCN9A, the gene that codes for Nav1.7 in humans, result in a congenital inability to sense pain and anosmia without affecting other sensations such as touch and temperature [20, 21]. Conversely, gain-of-function mutations lead to episodic pain such as primary erythromelalgia and paroxysmal extreme pain disorder [22, 23]. Despite an essential role of Navs in human pain regulation, little is known about their expression and function in native human sensory neurons. Recently, Han et al. showed that human Nav1.8 channels display slower inactivation kinetics and produce larger persistent current and ramp current than previously reported in other species [8]. In this study, we set out to compare the relative expression levels of different $\mathrm{Nav}$ subtypes in mouse DRG (mDRG) and hDRG tissues. We found that hDRG has a high ratio of Nav1.7 expression and low ratio of Nav1.8 expression compared to mDRG. To recapitulate chronic pain-induced changes in sodium channel expression, we treated dissociated hDRG neurons with paclitaxel, a chemotherapy agent that induces neuropathic pain [10]. Our data demonstrated that paclitaxel not only increased the expression of Nav1.7 but also enhanced the function of Nav1.7, as revealed by enlarged amplitude of sodium currents and increased firing frequency of action potentials, in human nociceptive neurons.

\section{Materials and Methods}

\section{Reagents}

Paclitaxel was purchased from Sigma (St. Louis, MO). Specific primers were designed using the Real-Time PCR web tool from IDT SciTools (Integrated DNA Technologies, Coralville, IA).

\section{Animals}

Adult CD1 mice (8-10 weeks old, both sexes) were obtained from Charles River Laboratories (Wilmington, MA) and used for PCR analysis. All the animal procedures performed in this study were approved by the Animal Care Committee of Duke University Medical Centre.

\section{Human DRG Tissues}

Human DRGs were obtained from healthy donors through the National Disease Research Interchange (Philadelphia,
PA) with permission from the Duke University Institutional Review Board. Postmortem L3-L5 DRGs were dissected from donors (see Table 1 for further details) and delivered in ice-cold culture medium to the laboratory at Duke University within $24-72 \mathrm{~h}$ of the donor's death.

\section{Immunohistochemistry}

Human DRGs were fixed in 4\% paraformaldehyde overnight and then sections $(12 \mu \mathrm{m})$ were cut on a cryostat. The sections were first blocked with $2 \%$ BSA for $1 \mathrm{~h}$ at room temperature, then incubated with peripherin (1:500, rabbit, Sigma) or a mixture of primary polyclonal TRPV1 antibody (1:400, rabbit, Neuromics, Edina, MN) and monoclonal NF200H antibody (1:1000, mouse, Sigma) overnight at $4{ }^{\circ} \mathrm{C}$. The sections were then incubated for $2 \mathrm{~h}$ at room temperature with cyanine 3 (Cy3)- and FITCconjugated secondary antibodies (1:400; Jackson ImmunoResearch, West Grove, PA). The DRG sections were then stained with DAPI (1:1000, Sigma, for $5 \mathrm{~min})$ and examined under a Nikon fluorescence microscope. Images were captured with a CCD Spot camera and analyzed with NIH Image J software or Adobe PhotoShop.

\section{Quantitative Real-Time RT-PCR}

DRG tissues were rapidly isolated in RNAse-free conditions. Total RNAs were extracted using an RNeasy Plus Mini kit (Qiagen, Germantown, MD). The quantity and quality of the eluted RNA samples were verified using a NanoDrop spectrophotometer (Thermo Fisher Scientific, Waltham, MA). Total RNAs $(0.5 \mu \mathrm{g})$ were reverse-

Table 1 Sex, age, and ethnicity of human DRG donors.

\begin{tabular}{llll}
\hline No. & Sex & Age (years) & Ethnicity \\
\hline 1 & Female & 76 & Caucasian \\
2 & Male & 67 & Caucasian \\
3 & Female & 66 & Caucasian \\
4 & Male & 66 & Caucasian \\
5 & Female & 58 & African-American \\
6 & Female & 55 & Caucasian \\
7 & Male & 54 & Caucasian \\
8 & Female & 50 & African-American \\
9 & Male & 49 & Caucasian \\
10 & Male & 31 & Caucasian \\
11 & Male & 29 & Caucasian \\
12 & Male & 27 & Caucasian \\
13 & Male & 55 & Caucasian \\
14 & Male & 41 & Caucasian \\
15 & Female & 27 & Caucasian \\
\hline
\end{tabular}


Table 2 Sequences of PCR primers for seven human Nav subtypes.

\begin{tabular}{lllrr}
\hline Gene & Forward & Reverse & Amplicon size & Accession number \\
\hline Nav1.1 & TCTCTTGCGGCTATTGAAAGAC & GGGCCATTTTCGTCGTCATCT & 86 & NM_001202435 \\
Nav1.2 & ATAGCGCTGTGGACTGCAATG & CTGTTTCAGTAGTTGTGCCCTCTG & 102 & NM_001040143 \\
Nav1.3 & CACCTTTAGCTGGGCTTTCCTG & CCAGCAGCACGTAATGTCAAC & 90 & NM_001081676 \\
Nav1.6 & CTGCGATCTTTCCGATTGCTC & AGGGCACCCACTGAATTTCC & 98 & NM_014191 \\
Nav1.7 & GGTTTCAGCACAGATTCAGGTC & CCAGCTGAAAGTGTCAAAGCTC & 102 & NM_002977 \\
Nav1.8 & CATCAAAGTGTCTGTCCACTCG & TTTCTCTGGAAGGTCAGTTCGG & 97 & NM_006514 \\
Nav1.9 & GGCACCGTTATCATCAACTGC & AAATCCCAGTGAAGACACACTC & 100 & NM_014139 \\
GAPDH & AGCCACATCGCTCAGACAC & GCCCAATACGACCAAATCC & 66 & NM_002046 \\
\hline
\end{tabular}

transcribed using the QuantiTect Reverse Transcription Kit according to the protocol of the manufacturer (Qiagen). The sequences of human Nav primers are listed in Table 2 (the sequences of mouse Nav primers were obtained from a previous publication [24]). We performed gene-specific mRNA analyses using the Bio-Rad CFX96 Real-Time RTPCR system (BioRad, Hercules, CA). Quantitative PCR amplification reactions contained the same amount of reverse transcription product: $7.5 \mu \mathrm{L}$ of Kapa Sybr ${ }^{\circledR}$ Fast Bio-Rad iCycler 2X qPCR Master Mix (Kapa Biosystems, Wilmington, MA) and $200 \mathrm{nmol} / \mathrm{L}$ forward and reverse primers in a final volume of $15 \mu \mathrm{L}$. The thermal cycling conditions were: $3 \mathrm{~min}$ of polymerase activation at $95{ }^{\circ} \mathrm{C}$, 45 cycles of denaturation at $95{ }^{\circ} \mathrm{C}$ for $10 \mathrm{~s}$, and annealing and extension at $60{ }^{\circ} \mathrm{C}$ for $30 \mathrm{~s}$, followed by a DNA melting curve for the determination of amplicon specificity. The expression level of the target mRNA was normalized to that of GAPDH mRNA and analyzed using the standard $2^{-\Delta \Delta C T}$ method. The relative mRNA expression of each Nav subtype is shown as a percentage of the total Navs (all seven subtypes) tested in DRGs [24].

\section{Human DRG Neuron Cultures}

Upon delivery, DRGs were rapidly dissected from the nerve roots, minced in $\mathrm{Ca}^{2+}$-free Hank's balanced salt solution (Thermo Fisher Scientific), and cultures were prepared as previously reported [11]. DRGs were digested at $37{ }^{\circ} \mathrm{C}$ in a humidified $\mathrm{CO}_{2}$ incubator for $120 \mathrm{~min}$ with collagenase Type II (Worthington, Lakewood, NJ; 290 units/mg, $12 \mathrm{mg} / \mathrm{mL}$ final concentration) and dispase II (Roche, Basel, Switzerland; 1 unit $/ \mathrm{mg}, 20 \mathrm{mg} / \mathrm{mL}$ ) in phosphate-buffered saline with $10 \mathrm{mmol} / \mathrm{L}$ HEPES, $\mathrm{pH}$ adjusted to 7.4 with $\mathrm{NaOH}$. hDRGs were mechanically dissociated using fire-polished pipettes, filtered through a $100-\mu \mathrm{m}$ nylon mesh, and centrifuged for $5 \mathrm{~min}(500 \mathrm{~g})$. The DRG cell pellet was re-suspended and plated on $0.5 \mathrm{mg} / \mathrm{mL}$ poly-D-lysine-coated glass coverslips. The DRG cultures were grown in Neurobasal medium supplemented with $10 \%$ fetal bovine serum, 2\% B-27 supplement, and $1 \%$ penicillin/streptomycin.

\section{Whole-Cell Patch-Clamp Recordings in Dissociated Human DRG Neurons}

Whole-cell patch-clamp recordings in small-diameter $(<50 \mu \mathrm{m})$ hDRG neurons were conducted at room temperature. We used patch pipettes to record transient $\mathrm{Na}^{+}$ currents and action potentials with an EPC10 amplifier (HEKA, Holliston, MA) and an Axopatch-200B amplifier with a Digidata 1440A (Axon Instruments, Sunnyvale CA). The patch pipettes were pulled from borosilicate capillaries (World Precision Instruments, Inc., Sarasota, FL). The resistance was 3-4 M $\Omega$ when filled with pipette solution. The recording chamber $(300 \mu \mathrm{L})$ was continuously superfused at 3-4 mL/min. Series resistance was compensated $(>80 \%)$ and leak subtraction was performed. Data were low-pass-filtered at $2 \mathrm{kHz}$ and sampled at $10 \mathrm{kHz}$. Patchmaster (HEKA) and pClamp10 (Axon Instruments) softwares were used during experiments and analysis. For recording $\mathrm{Na}^{+}$currents, the pipette solution contained (in mmol/L): $\mathrm{CsCl} 130, \mathrm{NaCl} 9, \mathrm{MgCl}_{2}$ 1, EGTA 10, HEPES 10 , adjusted to $\mathrm{pH} 7.3$ with $\mathrm{CsOH}$. For action potential recordings, the pipette solution contained (in $\mathrm{mmol} / \mathrm{L}$ ): K-gluconate 136, $\mathrm{NaCl} 10, \mathrm{MgCl}_{2}$ 1, EGTA 10, HEPES 10, Mg-ATP 2, adjusted to $\mathrm{pH} 7.3$ with $\mathrm{KOH}$. According to our preliminary study in hDRG neurons, action potentials were induced by $500 \mathrm{pA}$ current injection for $1 \mathrm{~s}$. The external solution for action potential recordings was composed of (in mmol/L): $\mathrm{NaCl} 140, \mathrm{KCl} 5, \mathrm{CaCl}_{2} 2, \mathrm{MgCl}_{2}$, HEPES 10, glucose 10 , adjusted to $\mathrm{pH} 7.3$ with $\mathrm{NaOH}$. The external solution for recording transient $\mathrm{Na}^{+}$currents contained (in mmol/L): $\mathrm{NaCl} 131$, TEACl 10, $\mathrm{CsCl} \mathrm{10,}$ $\mathrm{CaCl}_{2}$ 1, $\mathrm{MgCl}_{2} 2, \mathrm{CdCl}_{2}$ 0.3, 4-aminopyridine 3, HEPES 10 , glucose 10 , adjusted to $\mathrm{pH} 7.4$ with $\mathrm{NaOH}$. In voltageclamp experiments, transient $\mathrm{Na}^{+}$currents were evoked by a test pulse to $0 \mathrm{mV}$ from the holding potential $(-70 \mathrm{mV})$. 


\section{Statistical Analysis}

All data are expressed as mean \pm SEM. Biochemical and electrophysiological data were tested using one-way or twoway ANOVA followed by the post-hoc Bonferroni test or unpaired two-tailed Student's $t$-test. Pearson's correlation was used to test the linear dependence of two variables. Statistical analyses were performed with Prism 6.0 (GraphPad Software) and the criterion for statistical significance was $P<0.05$.

\section{Results}

\section{Immunohistochemical Characterization of Human DRG Neurons}

Immunofluorescent staining of hDRGs is a challenge, because of the high background of autofluorescence in human tissue [25]. We were able to obtain intact frozen sections of hDRGs, as demonstrated by DAPI staining, which shows all the nuclei in sections (Fig. 1A). Notably, hDRG neurons were much larger than rodent DRG neurons, and small hDRG neurons were identified with diameters of $<50 \mu \mathrm{m}[7,11]$. Neurofilament (NF200) is a marker for myelinated A-fiber neurons in rodent DRGs [10]. However, in hDRG sections, NF200 labeled both large and small neurons (Fig. 1B). Double-staining revealed co-localization of NF200 (NF200H) with TRPV1, a marker for C-fiber neurons in the rodent DRG [26] (Fig. 1C, D). Peripherin is another marker for small nociceptor neurons in the rodent DRG [27]. Consistently, peripherin was also expressed by small-diameter hDRG neurons (Fig. 1E). Limited size analysis showed that the average diameters of TRPV1- and peripherin-positive hDRG neurons were $44 \pm 7$ and $38 \pm 7 \mu \mathrm{m}$, respectively ( $n=28-37$ neurons/group). Our results are in agreement
Fig. 1 Histochemical characterization of human DRG neurons. A DAPI nuclear staining in an intact hDRG section. B, C Double-staining of neurofilament (NF200 or NFH, green, B) and TRPV1 (red, C) in the section. D Merged image of DAPI, NFH, and TRPV1 staining. E Immunostaining of peripherin in an hDRG section. Scale bar, $100 \mu \mathrm{m}$ for $\mathbf{A}-$ E.
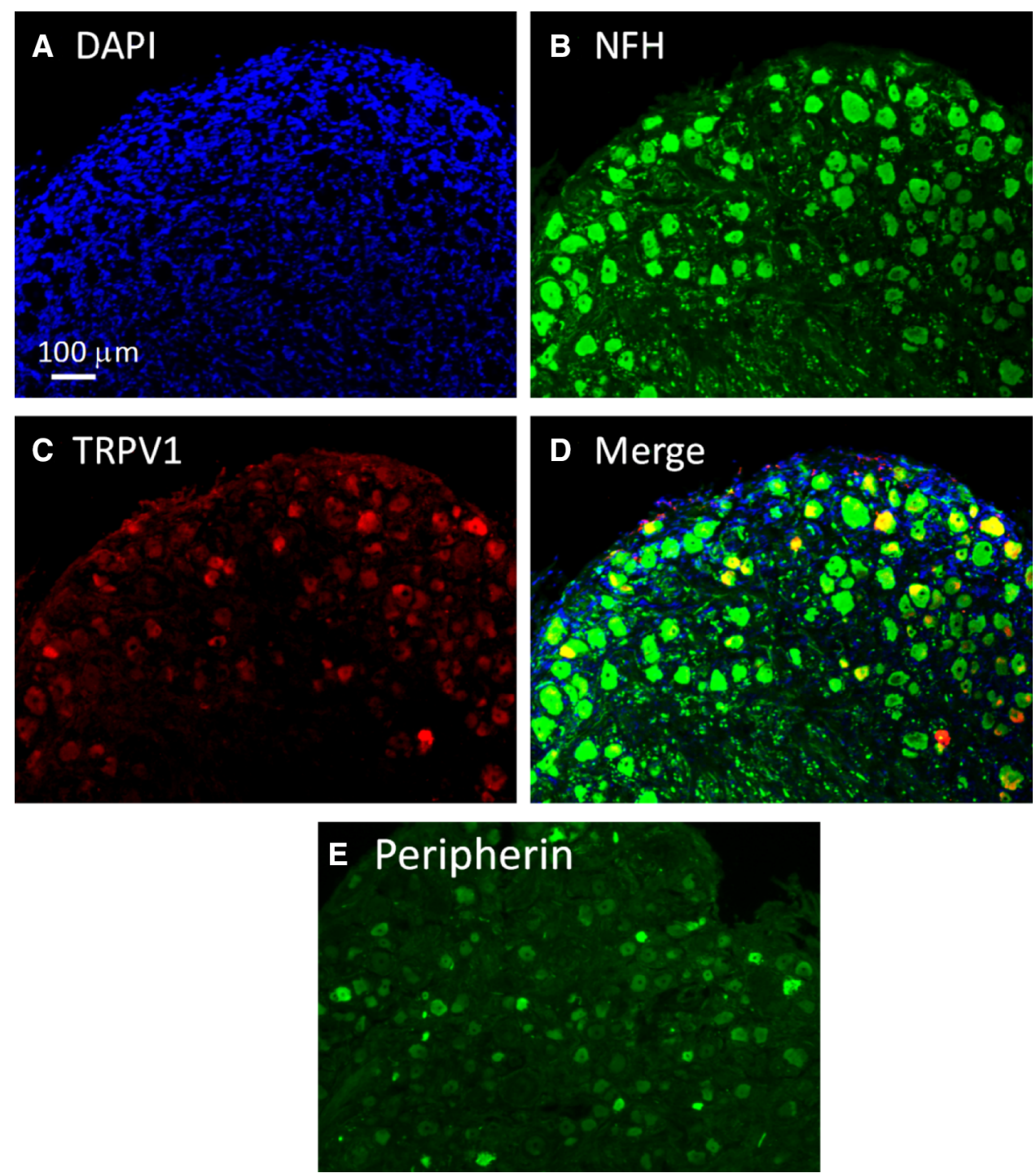
with previous reports that hDRG neurons with diameters $<50 \mu \mathrm{m}$ can be regarded as small nociceptive neurons $[7,11]$.

\section{Distinct Expression of Sodium Channel Subtypes in Human and Mouse DRG Tissues}

We used quantitative real-time PCR (qPCR) to compare the transcriptional expression of seven Nav subtypes in hDRGs and mDRGs: TTX-sensitive Navs (Nav1.1, Nav1.2, Nav.1.3, Nav1.6, and Nav1.7) and TTX-resistant Navs (Nav1.8 and Nav1.9). Notably, all these subtypes are known to be expressed in rodent DRG neurons [15, 16, 28]. To define the relative expression levels of each subtype in mouse and human DRG tissues, we calculated the percentage expression of each subtype based on the total expression of all these subtypes (Fig. 2A,B). Striking species differences were found in Nav1.1, Nav1.6, Nav1.7, Nav1.8, and Nav1.9 (Table 3). The ratio of Nav1.7 expression in the hDRG $(49.2 \pm 5.6 \%)$ was much higher than that in the mDRG $(18.2 \pm 1.7 \%)$. On the other hand, the mDRG had a much higher ratio of Nav1.8 (44.9 $\pm 2.4 \%)$ than that of the hDRG $(12.1 \pm 2.1 \%)$. Also, the ratio of Nav1.6 was higher in the hDRG $(8.8 \pm 0.8 \%)$ than in the $\operatorname{mDRG}(3.3 \pm 0.3 \%)$ (Fig. 2A, B).

\section{Sodium Channel Subtype Expression in Human DRGs Shows No Sex and Age Difference}

We also investigated the sex differences in Nav subtype expression in $\mathrm{hDRG}$ and $\mathrm{mDRG}$ tissues. The relative expression of each Nav subtype in the hDRGs of 6 men and 6 women showed no sex difference in the expression of any subtype (Fig. 2A). Similarly, their relative expression in the mDRGs of 6 males and 6 females showed no sex difference in the expression of any mouse Nav subtype (Fig. 2B). Further comparison of the relative expression of Nav1.7 in hDRGs also revealed no statistical differences between men and women (Fig. 3A). We also analyzed Nav1.7 expression in hDRG donors of different ages, ranging from 27 to 76 years and found that although the expression was slightly lower in older donors, there was no significant correlation between Nav1.7 expression and age (Fig. 3B).

Table 3 Statistics of species difference in the expression of mouse and human Nav1.1, Nav1.2, Nav1.3, Nav1.6, Nav1.7, Nav1.8, and Nav1.9 from the samples shown in Fig. 2.

\begin{tabular}{lclr}
\hline Gene & Mean difference & $95.00 \%$ CI of diff. & $P$ value \\
\hline Nav1.1 & -8.85 & -13.52 to -4.179 & $<0.0001$ \\
Nav1.2 & 1.411 & -3.261 to 6.082 & $>0.9999$ \\
Nav1.3 & -1.996 & -6.667 to 2.676 & $>0.9999$ \\
Nav1.6 & 6.306 & 1.634 to 10.98 & 0.0022 \\
Nav1.7 & 31.88 & 27.21 to 36.55 & $<0.0001$ \\
Nav1.8 & -33.68 & -38.35 to -29 & $<0.0001$ \\
Nav1.9 & 4.841 & 0.1695 to 9.512 & 0.0374 \\
\hline
\end{tabular}

$N=12$ samples/group; two-way ANOVA followed by post-hoc Bonferroni test.

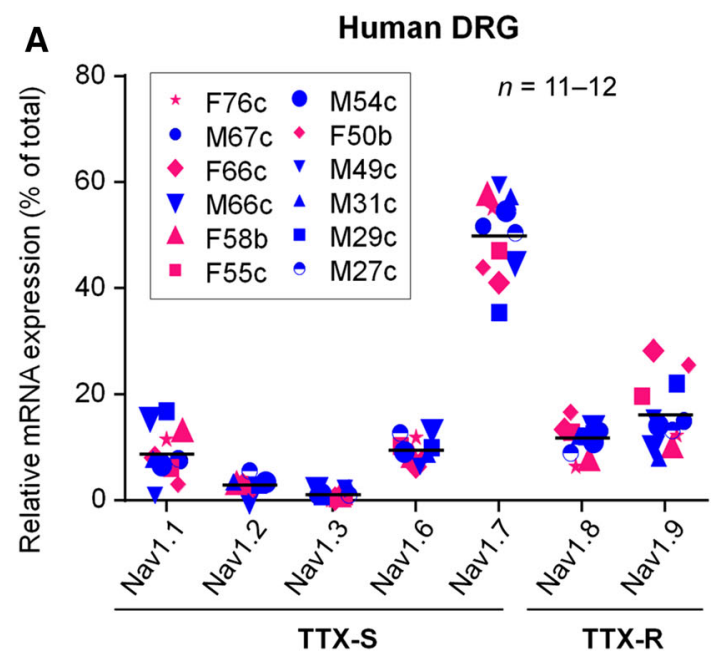

Fig. 2 Distinct expression of sodium channel subtypes in human and mouse DRG tissues. A, B qPCR showing the relative percentage expression of TTX-sensitive Nav subtypes (TTX-S: Nav1.1, Nav1.2, Nav.1.3, Nav1.6, and Nav1.7) and TTX-resistant subtypes (TTX-R: Nav1.8 and Nav1.9) in hDRG (A) and mDRG (B) tissues. The

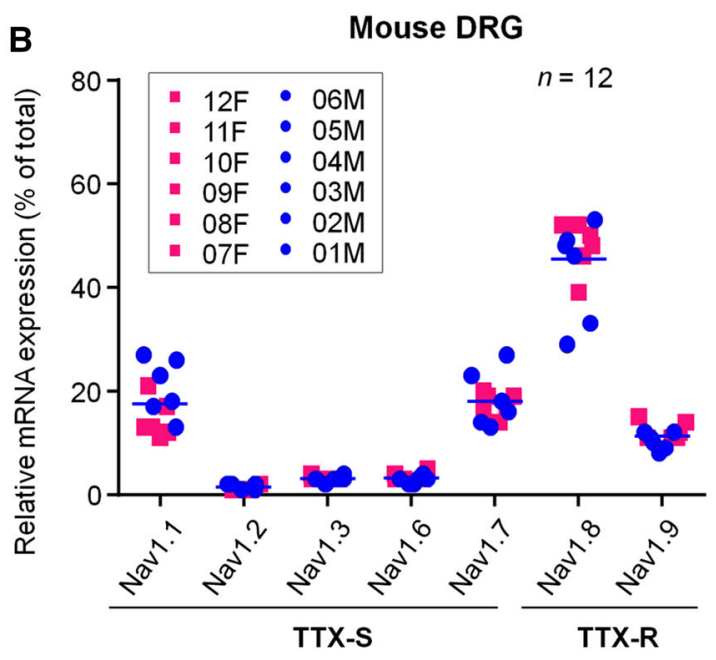

percentage expression of each subtype is based on the total expression of all seven subtypes. Note the species differences in Nav1.6, Nav1.7, and Nav1.8 expression in hDRGs and mDRGs $(n=12$ samples/group; blue, male samples; red, female samples; b, African_American; c, Caucasian; F, female; M, male. 


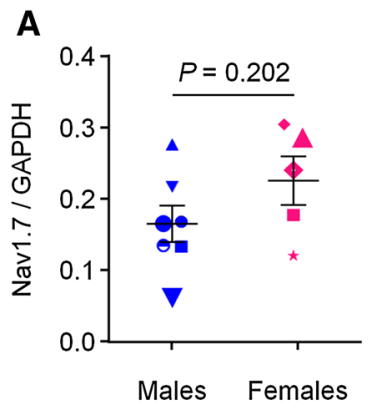

Fig. 3 qPCR showing lack of sex and age differences in Nav 1.7 expression in human DRGs. A Comparison of Nav1.7 mRNA expression in male (blue) and female (red) hDRG tissues. Note the absence of a statistical difference $(P=0.202$, unpaired Student's $t$ test, $n=6$ donors/group). B No correlation between age and Nav1.7

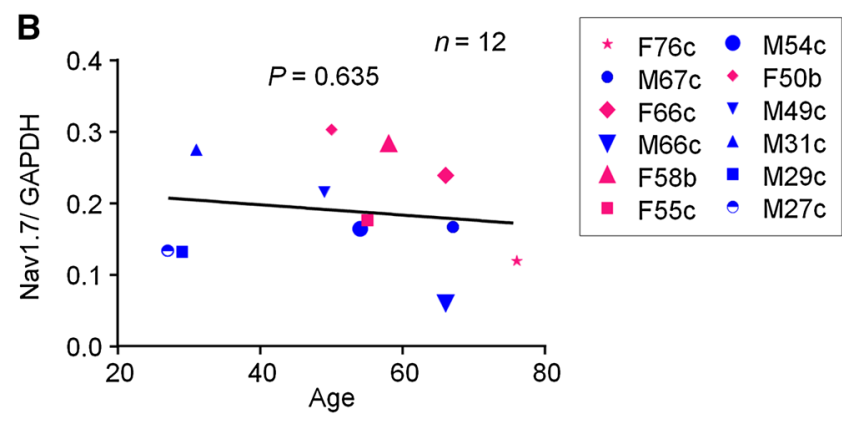

expression in hDRGs $(P=0.635, n=12$ donors, Pearson's correlation). GAPDH was used as a positive control, and the value of Nav1.7 expression was normalized to that of GAPDH. All data are expressed as mean \pm SEM.
Fig. 4 Paclitaxel increases Nav1.7 but not Nav1.8 expression in human DRGs. A, B qPCR showing Nav1.7 mRNA (A) and Nav1.8 mRNA (B) levels in hDRGs $24 \mathrm{~h}$ after paclitaxel treatment $(0.1$ and $1 \mu \mathrm{mol} / \mathrm{L}) . * * P<0.01$ $* * * P<0.001$, one-way ANOVA, $n=6-9$ cultures/group. All the data are expressed as mean $\pm \mathrm{SEM}$.
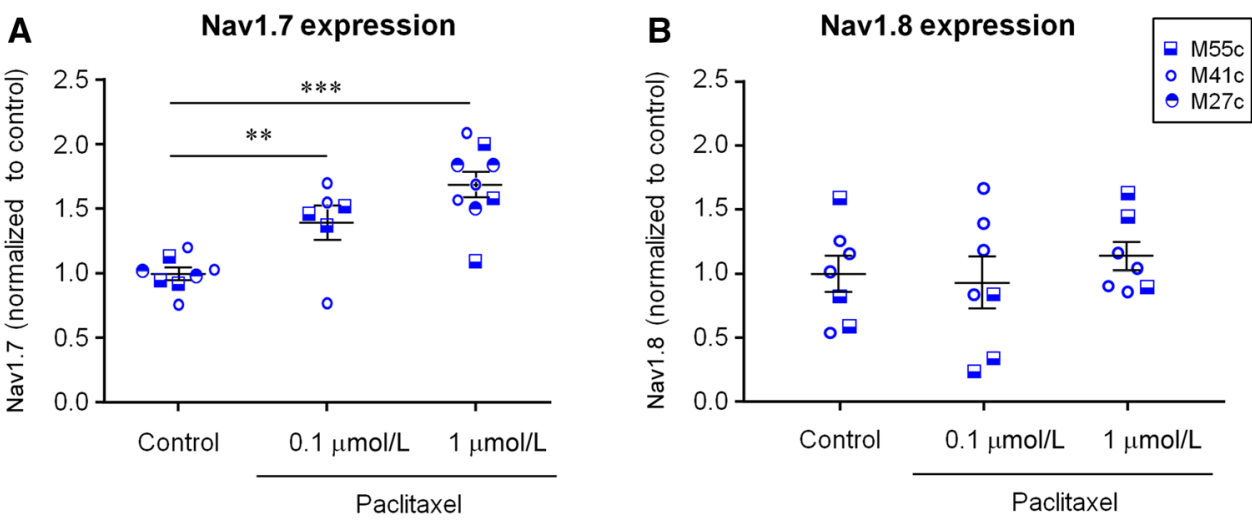

\section{Paclitaxel Increases Nav1.7 Expression, Transient Sodium Currents, and Action Potential Firing Frequency in hDRG Neurons}

Previous studies have shown that Nav1.7 is upregulated in DRG neurons under chronic pain conditions such as diabetic neuropathy, and contributes to the pathogenesis of neuropathic pain [18, 29, 30]. Paclitaxel is a widely-used chemotherapeutic drug that induces neuropathy and neuropathic pain [10, 31]. To mimic chronic pain in a dish, we treated dissociated hDRG neurons with paclitaxel $(0.1$ and $1 \mu \mathrm{mol} / \mathrm{L})$ for $24 \mathrm{~h}$. qPCR analysis revealed a significant and dose-dependent increase in Nav1.7 mRNA expression after the treatment (Fig. 4A). By contrast, Nav1.8 mRNA expression in hDRG cultures did not change after this treatment (Fig. 4B).

To assess the function of Navs including Nav1.7 in hDRG neurons, we recorded transient $\mathrm{Na}^{+}$currents in small-diameter neurons $(<50 \mu \mathrm{m})$. In untreated $\mathrm{hDRG}$ neurons, the amplitude of transient $\mathrm{Na}^{+}$currents was $3131 \pm 573.1 \mathrm{pA}(n=29$ neurons from 3 DRGs/3 donors, Fig. 5A, B). Notably, paclitaxel ( $1 \mu \mathrm{mol} / \mathrm{L}, 24 \mathrm{~h})$ caused a significant increase in the amplitude of transient $\mathrm{Na}^{+}$ currents (4853 \pm 634.4 pA: Fig. $5 B)$.
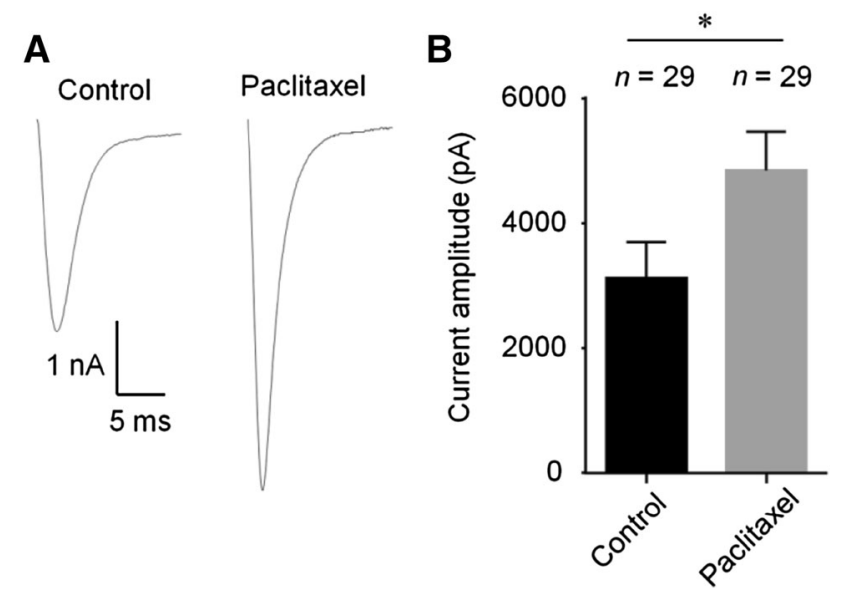

Fig. 5 Paclitaxel increases transient sodium currents in dissociated human DRG neurons. (A) Traces of transient $\mathrm{Na}^{+}$currents in small hDRG neurons with diameters $<50 \mu \mathrm{m}$. Note that the current was greater after paclitaxel treatment $(1 \mu \mathrm{mol} / \mathrm{L}, 24 \mathrm{~h})$. (B) Quantification of the $\mathrm{Na}^{+}$current amplitude. $* P<0.05$, unpaired Student's $t$-test, $n=29$ neurons from 3 DRGs/3 donors. All data are expressed as mean \pm SEM.

Finally, we investigated whether paclitaxel changes the excitability of human nociceptors by recording action potentials in small hDRG neurons. The rheobase for 
inducing action potentials was $285.7 \pm 59.5$ pA $(n=7$ neurons) in untreated $\mathrm{hDRG}$ neurons. After paclitaxel treatment $(1 \mu \mathrm{mol} / \mathrm{L}, 24 \mathrm{~h})$, there was a slight decrease in rheobase $(216.7 \pm 66.67 \mathrm{pA}, n=3$ neurons, $P>0.05$, unpaired two-tailed Student's $t$-test). This lack of significance may result from the small number of neurons recorded. Current-clamp recording revealed that current injection (500 pA, $1 \mathrm{~s}$ ) evoked action potentials in hDRG neurons (Fig. 6A). In untreated hDRG neurons, the firing frequency was $5.41 \pm 0.74 \mathrm{~Hz}$ (Fig. 6B). Notably, paclitaxel $(1 \mu \mathrm{mol} / \mathrm{L}, 24 \mathrm{~h})$ caused a significant increase in firing frequency $(8.71 \pm 1.10 \mathrm{~Hz}$ : Fig. $6 \mathrm{~B})$. This result suggests a possible increase in the excitability of hDRG neurons after paclitaxel treatment. However, additional features of neuronal excitability such as resting potential, membrane resistance, and threshold current need to be tested in future studies.

\section{Discussion}

There have been many failures in developing new analgesics, because (1) molecular mechanisms identified in rodents may not apply to humans and (2) animal models of pain may not represent human pains $[5,6]$. To fill in this translational gap, several groups including our own have begun to test pain mechanisms in native hDRG neurons obtained from human donors [7-11]. These studies reveal that peripheral mechanisms of pain are likely to be shared by mice and humans and demonstrate a translational research strategy to improve the preclinical validation of novel analgesics. We have recently demonstrated that the autism gene SHANK3 is expressed by both mouse and human DRG neurons and regulates TRPV1 function in the
A
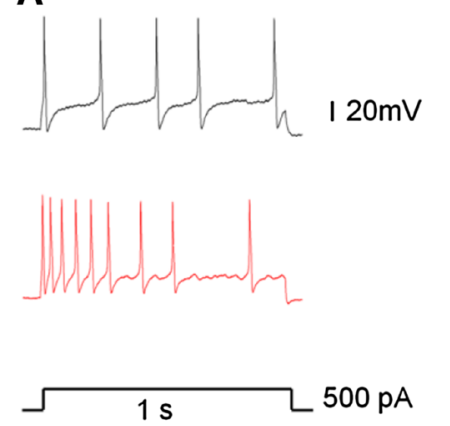

B

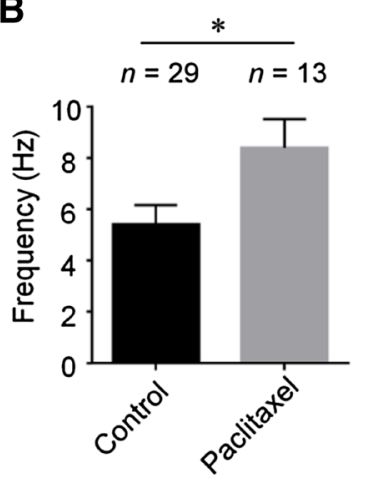

Fig. 6 Paclitaxel increases the action potential firing frequency in human DRG neurons. A Traces of action potentials in small hDRG neurons with diameters $<50 \mu \mathrm{m}$. Note that the firing frequency increased after paclitaxel treatment ( $1 \mu \mathrm{mol} / \mathrm{L}, 24 \mathrm{~h})$. B Quantification of the firing frequency of action potentials. $* P<0.05$, unpaired Student's $t$-test, $n=13$ and 29 neurons/group, from 3 DRGs/3 donors. All data are expressed as mean \pm SEM.
DRG neurons of both species, offering a novel peripheral mechanism of pain dysregulation in autism [11].

Although the role of human Nav1.7 has been wellstudied in heterologous cells and rodent DRG neurons by over-expression of different human Nav1.7 mutants in these cells, the specific role of Nav1.7 in native hDRG neurons is unclear. Global Nav1.7-knockout mice recapitulate the phenotype of human congenital indifference to pain [32]. Nerve trauma after diabetes and spared nerve injury have been shown to cause upregulation of Nav1.7 in mDRG neurons and redistribution of Nav1.7 channels toward peripheral axons, leading to enhanced neuropathic pain in mice [29, 30]. However, it has also been shown that pain induced by the chemotherapeutic agent oxaliplatin and cancer-induced bone pain may not require the presence of Nav1.7 channels [33]. Furthermore, endogenous opioids appear to contribute to pain insensitivity in mice lacking Nav1.7 [34]. Thus, there could be compensatory regulations in mice lacking Nav1.7, emphasizing the importance of studying Nav1.7 in native hDRG neurons.

Our data have shown striking species differences in the expression of different Nav subtypes. Especially, the ratio of Nav1.7 expression in the hDRG $(50 \%)$ is much higher than in the mDRG (20\%). On the other hand, the mDRG has a much higher ratio of Nav1.8 (45\%) than the hDRG (15\%), and a critical role of Nav1.8 in the pathogenesis of neuropathic pain has been well documented in rodents $[35,36]$. It is reasonable to postulate that the high ratio of expression of Nav1.7 in hDRG neurons is associated with an enhanced role of this subtype in human pain conditions compared with other subtypes. This notion is supported by human genetics showing that both most pain-related gainof-function and loss-of-function mutations are found in SCN9A [21-23], while fewer mutations have been found in SCN10A (encoding Nav1.8) [8], SCN11A (encoding Nav1.9) [37], and SCN8A (encoding Nav1.6) [38]. Inherited erythromelalgia (IEM), a severe pain syndrome characterized by episodes of intense burning pain triggered by warmth, is caused by mutations in Nav1.7 in sensory and sympathetic neurons. A newly-identified IEM mutation (Nav1.7-A1632G) in a multi-generation family is responsible for hyperexcitability in sensory neurons, and the increased responses to temperature also suggest a cellular basis for the warmth-triggered pain in IEM [39]. It is noteworthy that an hDRG neuron survived for 76 years in our oldest donor (Table 1), whereas a rodent DRG neuron or a heterologous cell only survives for a few days to a few months. Thus, Nav1.7 in native hDRG neurons could be regulated by different mechanisms. Interestingly, we did not find sex- and age-dependent changes in Nav1.7 expression in hDRG neurons, suggesting that maintaining homeostasis of its expression is important for this critical ion channel. However, paclitaxel, which is known to 
induce neuropathy, caused a marked increase in Nav1.7 expression, associated with increases in the transient $\mathrm{Na}^{+}$ current and excitability (as revealed by increased action potential firing), in small-diameter (presumably nociceptive) hDRG neurons. Although other Nav subtypes could also contribute to the transient $\mathrm{Na}^{+}$current, Nav1.7 is a major contributor to this TTX-sensitive current $[18,32]$.

In summary, naïve $\mathrm{hDRG}$ neurons provide a translational model in which to study voltage-gated $\mathrm{Na}^{+}$ channels such as Nav1.7 and "human pain in a dish". hDRG neurons have been used to study acute inflammatory pain mechanisms following stimulation by inflammatory mediators such as prostaglandin E2 [7], but they can also be used to investigate neuropathic pain mechanisms after paclitaxel treatment as demonstrated in this study. In future studies, we will try to collect hDRGs from donors with chronic pain conditions to investigate the regulation of Nav subtypes in human pain. We will also conduct single-cell analysis to characterize the expression of Nav subtypes in different populations of hDRG neurons, as shown in mDRG neurons [40, 41]. It is noteworthy that paclitaxel did not change Nav1.8 expression in hDRG neurons, in support of a previous report that Nav1.8 does not contribute to chemotherapy-induced mechanical allodynia in rats [42]. Thus, naive hDRG neurons can be used to test new pain therapeutics such as Nav1.7 inhibitors, as the last-step of target validation before the initiation of clinical trials.

Acknowledgements This work was supported in part by NIH RO1 Grants NS87988, DE17794, and DE22743 to R.R.J and NS89479 to S.Y.L and R.R.J.

Open Access This article is distributed under the terms of the Creative Commons Attribution 4.0 International License (http:// creativecommons.org/licenses/by/4.0/), which permits unrestricted use, distribution, and reproduction in any medium, provided you give appropriate credit to the original author(s) and the source, provide a link to the Creative Commons license, and indicate if changes were made.

\section{References}

1. Gold MS, Gebhart GF. Nociceptor sensitization in pain pathogenesis. Nat Med 2010, 16: 1248-1257.

2. Ji RR, Chamessian A, Zhang YQ. Pain regulation by nonneuronal cells and inflammation. Science 2016, 354: 572-577.

3. Zhou LJ, Liu XG. Glial activation, a common mechanism underlying spinal synaptic plasticity? Neurosci Bull 2017, 33 : 121-123.

4. Liu T, Gao YJ, Ji RR. Emerging role of Toll-like receptors in the control of pain and itch. Neurosci Bull 2012, 28: 131-144.

5. Woolf CJ. Overcoming obstacles to developing new analgesics. Nat Med 2010, 16: 1241-1247.

6. Mogil JS. Animal models of pain: progress and challenges. Nat Rev Neurosci 2009, 10: 283-294.
7. Davidson S, Copits BA, Zhang J, Page G, Ghetti A, Gereau RW. Human sensory neurons: membrane properties and sensitization by inflammatory mediators. Pain 2014, 155: 1861-1870.

8. Han C, Estacion M, Huang J, Vasylyev D, Zhao P, Dib-Hajj SD, et al. Human $\mathrm{Na}(\mathrm{v}) 1.8$ : enhanced persistent and ramp currents contribute to distinct firing properties of human DRG neurons. J Neurophysiol 2015, 113: 3172-3185.

9. Li Y, Adamek P, Zhang H, Tatsui CE, Rhines LD, Mrozkova P, et al. The cancer chemotherapeutic paclitaxel increases human and rodent sensory neuron responses to TRPV1 by activation of TLR4. J Neurosci 2015, 35: 13487-13500.

10. Xu ZZ, Kim YH, Bang S, Zhang Y, Berta T, Wang F, et al. Inhibition of mechanical allodynia in neuropathic pain by TLR5mediated A-fiber blockade. Nat Med 2015, 21:1326-1331.

11. Han Q, Kim YH, Wang X, Liu D, Zhang ZJ, Bey AL, et al. SHANK3 deficiency impairs heat hyperalgesia and TRPV1 signaling in primary sensory neurons. Neuron 2016, 92: 1279-1293.

12. Cummins TR, Dib-Hajj SD, Black JA, Waxman SG. Sodium channels and the molecular pathophysiology of pain. Prog Brain Res 2000, 129: 3-19.

13. Bennett DL, Woods CG. Painful and painless channelopathies. Lancet Neurol 2014, 13: 587-599.

14. Jiang F, Hua LM, Jiao YL, Ye P, Fu J, Cheng ZJ, et al. Activation of mammalian target of rapamycin contributes to pain nociception induced in rats by $\mathrm{BmK} \mathrm{I}$, a sodium channel-specific modulator. Neurosci Bull 2014, 30: 21-32.

15. Waxman SG, Dib-Hajj S, Cummins TR, Black JA. Sodium channels and pain. Proc Natl Acad Sci USA 1999, 96: $7635-7639$.

16. Bao L. Trafficking regulates the subcellular distribution of voltage-gated sodium channels in primary sensory neurons. Mol Pain 2015, 11: 61 .

17. Pan HL, Liu BL, Lin W, Zhang YQ. Modulation of Nav1.8 by lysophosphatidic acid in the induction of bone cancer pain. Neurosci Bull 2016, 32: 445-454.

18. Lee JH, Park CK, Chen G, Han Q, Xie RG, Liu T, et al. A monoclonal antibody that targets a NaV1.7 channel voltage sensor for pain and itch relief. Cell 2014, 157: 1393-1404.

19. Amaya F, Wang H, Costigan M, Allchorne AJ, Hatcher JP, Egerton J, et al. The voltage-gated sodium channel $\mathrm{Na}(\mathrm{v}) 1.9$ is an effector of peripheral inflammatory pain hypersensitivity. J Neurosci 2006, 26: 12852-12860.

20. Cox JJ, Reimann F, Nicholas AK, Thornton G, Roberts E, Springell K, et al. An SCN9A channelopathy causes congenital inability to experience pain. Nature 2006, 444: 894-898.

21. Weiss J, Pyrski M, Jacobi E, Bufe B, Willnecker V, Schick B, et al. Loss-of-function mutations in sodium channel Nav1.7 cause anosmia. Nature 2011, 472: 186-190.

22. Drenth JP, Finley WH, Breedveld GJ, Testers L, Michiels JJ, Guillet $\mathrm{G}$, et al. The primary erythermalgia-susceptibility gene is located on chromosome 2q31-32. Am J Hum Genet 2001, 68: 1277-1282.

23. Fertleman CR, Baker MD, Parker KA, Moffatt S, Elmslie FV, Abrahamsen B, et al. SCN9A mutations in paroxysmal extreme pain disorder: allelic variants underlie distinct channel defects and phenotypes. Neuron 2006, 52: 767-774.

24. Laedermann CJ, Pertin M, Suter MR, Decosterd I. Voltage-gated sodium channel expression in mouse DRG after SNI leads to reevaluation of projections of injured fibers. Mol Pain 2014, 10: 19.

25. Davidson S, Golden JP, Copits BA, Ray PR, Vogt SK, Valtcheva $\mathrm{MV}$, et al. Group II mGluRs suppress hyperexcitability in mouse and human nociceptors. Pain 2016, 157: 2081-2088.

26. Tominaga M, Caterina MJ, Malmberg AB, Rosen TA, Gilbert H, Skinner $\mathrm{K}$, et al. The cloned capsaicin receptor integrates multiple pain-producing stimuli. Neuron 1998, 21: 531-543. 
27. Amaya F, Decosterd I, Samad TA, Plumpton C, Tate S, Mannion $\mathrm{RJ}$, et al. Diversity of expression of the sensory neuron-specific TTX-resistant voltage-gated sodium ion channels SNS and SNS2. Mol Cell Neurosci 2000, 15: 331-342.

28. Xie W, Strong JA, Ye L, Mao JX, Zhang JM. Knockdown of sodium channel NaV1.6 blocks mechanical pain and abnormal bursting activity of afferent neurons in inflamed sensory ganglia. Pain 2013, 154: 1170-1180.

29. Chattopadhyay M, Mata M, Fink DJ. Continuous delta-opioid receptor activation reduces neuronal voltage-gated sodium channel (NaV1.7) levels through activation of protein kinase $\mathrm{C}$ in painful diabetic neuropathy. J Neurosci 2008, 28: 6652-6658.

30. Laedermann CJ, Cachemaille M, Kirschmann G, Pertin M, Gosselin RD, Chang I, et al. Dysregulation of voltage-gated sodium channels by ubiquitin ligase NEDD4-2 in neuropathic pain. J Clin Invest 2013, 123: 3002-3013.

31. Li Y, Zhang H, Zhang H, Kosturakis AK, Jawad AB, Dougherty PM. Toll-like receptor 4 signaling contributes to paclitaxelinduced peripheral neuropathy. J Pain 2014, 15: 712-725.

32. Gingras J, Smith S, Matson DJ, Johnson D, Nye K, Couture L, et al. Global Nav1.7 knockout mice recapitulate the phenotype of human congenital indifference to pain. PLoS One 2014, 9: e105895.

33. Minett MS, Falk S, Santana-Varela S, Bogdanov YD, Nassar MA, Heegaard AM, et al. Pain without nociceptors? Nav1.7independent pain mechanisms. Cell Rep 2014, 6: 301-312.

34. Minett MS, Pereira V, Sikandar S, Matsuyama A, Lolignier S, Kanellopoulos AH, et al. Endogenous opioids contribute to insensitivity to pain in humans and mice lacking sodium channel Nav1.7. Nat Commun 2015, 6: 8967.
35. Gold MS, Weinreich D, Kim CS, Wang R, Treanor J, Porreca F, et al. Redistribution of $\mathrm{Na}(\mathrm{V}) 1.8$ in uninjured axons enables neuropathic pain. J Neurosci 2003, 23: 158-166.

36. Porreca F, Lai J, Bian D, Wegert S, Ossipov MH, Eglen RM, et al. A comparison of the potential role of the tetrodotoxininsensitive sodium channels, PN3/SNS and NaN/SNS2, in rat models of chronic pain. Proc Natl Acad Sci USA 1999, 96: 7640-7644.

37. Dib-Hajj SD, Black JA, Waxman SG. NaV1.9: a sodium channel linked to human pain. Nat Rev Neurosci 2015, 16: 511-519.

38. Tanaka BS, Zhao P, Dib-Hajj FB, Morisset V, Tate S, Waxman $\mathrm{SG}$, et al. A gain-of-function mutation in Nav1.6 in a case of trigeminal neuralgia. Mol Med 2016, 22. doi:10.2119/molmed. 2016.00131.

39. Yang Y, Huang J, Mis MA, Estacion M, Macala L, Shah P, et al. Nav1.7-A1632G mutation from a family with inherited erythromelalgia: enhanced firing of dorsal root ganglia neurons evoked by thermal stimuli. J Neurosci 2016, 36: 7511-7522.

40. Usoskin D, Furlan A, Islam S, Abdo H, Lonnerberg P, Lou D, et al. Unbiased classification of sensory neuron types by largescale single-cell RNA sequencing. Nat Neurosci 2015, 18: $145-153$.

41. Li CL, Li KC, Wu D, Chen $\mathrm{Y}$, Luo $\mathrm{H}$, Zhao JR, et al. Somatosensory neuron types identified by high-coverage singlecell RNA-sequencing and functional heterogeneity. Cell Res 2016, 26: 967.

42. Joshi SK, Mikusa JP, Hernandez G, Baker S, Shieh CC, Neelands $\mathrm{T}$ et al. Involvement of the TTX-resistant sodium channel Nav 1.8 in inflammatory and neuropathic, but not post-operative, pain states. Pain 2006, 123: 75-82. 